\title{
Broken ergodicity and glassy behavior in a deterministic chaotic map
}

\author{
A. Crisanti, M. Falcioni $(a)$ and A. Vulpiani $(a)$ \\ Dipartimento di Fisica, Università "La Sapienza", P.le A. Moro 2, 00185, Roma, Italy
}

(May 1, 2019)

\begin{abstract}
A network of $N$ elements is studied in terms of a deterministic globally coupled map which can be chaotic. There exists a range of values for the parameters of the map where the number of different macroscopic configurations $\mathcal{N}(N)$ is very large, $\mathcal{N}(N) \sim \exp \sqrt{c(a) N}$ and there is violation of selfaveraging. The time averages of functions, which depend on a single element, computed over a time $T$, have probability distributions that do not collapse to a delta function, for increasing $T$ and $N$. This happens for both chaotic and regular motion, i.e. positive or negative Lyapunov exponent.
\end{abstract}

05.45. $+\mathrm{b}, 05.90 .+\mathrm{m}$

Glassy behavior is still one of the central subjects of modern physics [1]. Among the most interesting features are the violation of time traslation invariance, and fluctuation-dissipation theorem, even for very long times [2]. This follows from the breaking of ergodicity in the phase space caused by a rough energy landscape. In spin glasses, the paradigmatic system, the complex dynamics follows from frustration and randomness. It is, however, obvious that these are not a prerequisite for glassy behavior, think for example to ordinary window glass. Recently an increasing amount of work has been devoted to the study of glassy behavior in non random systems [3]. In this case the complex behavior follows from the existence of a large number of metastable states which slows down the dynamics.

We shall concentrate on systems of globally coupled elements without any intrinsic disorder. Dynamical systems of this type have been studied recently, in great details, in many fields [4], and used to model a wide class of phenomena, such as Josephson-junction array, evolution dynamic of ecosystems, neural-networks for neurodynamics.

The interesting point is that the macroscopic description of these systems may be extremely rich. The variety of the macroscopic description can be taken as an indication of complexity [5].

In this Letter we shall study a network of elements a globally coupled map - given by:

$$
x_{i}(n+1)=(1-\epsilon) f\left(x_{i}(n)\right)+\frac{\epsilon}{N} \sum_{j=1}^{N} f\left(x_{j}(n)\right)
$$

where $n$ is the discrete time variable and $i=1, \cdots, N$ is the index identifying the elements. For $f(x)$ we choose the logistic map

$$
f(x)=1-a x^{2} .
$$

The system (11), that has been thoroughly studied by Kaneko [6], displays a very rich behavior. Interestingly, for certain values of the parameters $a$ and $\epsilon$, one observes a violation of the law of large numbers: a subtle coherence among the elements $x_{i}$ induces anomalous fluctuations of the mean field

$$
h(n)=\frac{1}{N} \sum_{j=1}^{N} f\left(x_{j}(n)\right)
$$

so that the mean square deviation, $\left\langle h^{2}\right\rangle-\langle h\rangle^{2}$, decays as $N^{-\beta}$, with $\beta<1$. Moreover, the elements split into clusters whose probability distributions resembles, for suitable values of the parameter, to those derived from the theory of spin glasses [7.

In this letter we show that the analogy of this system with spin glasses, previously proposed by Kaneko, is very deep: for a certain range of values of the control parameter $a$, the system exhibits a very large numbers of "macroscopic" states and a violation of selfaveraging, indicating that the system is "complex" in the above stated sense.

Because of the global coupling in (1), one can have the phenomenon of clusterization: if, at a certain time $\widetilde{n}$, the variables of some elements take on identical values, they will maintain equal values for every $n>\widetilde{n}$. Therefore, it is possible to introduce a "macroscopic" description of the system in terms of the number $N_{i}$ of elements in the $i^{\text {th }}$ cluster and to identify a macroscopic state by the set of integers $\left\{N_{i}\right\}=\left[N_{1}, N_{2}, \ldots, N_{m} \mid \sum_{i=1}^{m} N_{i}=N\right]$, that characterize the population of each cluster. All the microscopic states with the same cluster structure, i.e. with identical values for the $\left\{N_{i}\right\}$, but differing either by a permutation of some $x_{j}$ or by the value of the variables $x_{j}$ in a particular cluster, belong to the same macroscopic state (by definition).

We start by estimating the number of relevant macroscopic states of the system as a function of $N$, for different values of $a$. This is done by generating for each value of $N$ and $a$ a set $\mathcal{M} \gg 1$ of initial conditions $x_{i}^{(\alpha)}(0)$, with $\alpha=1,2, \cdots, \mathcal{M}$, selecting at random each component $x_{i}^{(\alpha)}(0)$ with uniform distribution in the interval $[-1,+1]$; we used $\mathcal{M}=10^{4} \div 5 \times 10^{4}$. The numerical 
results reported here were obtained with $\epsilon=0.1$. For each initial condition the map (11) is iterated and the final "cluster structure" recorded. In all cases we have discarded $6 \cdot 10^{4}$ thermalization steps before performing measurements. From each set of initial conditions we obtained a "cluster structure", i.e. a set $\left\{N_{j}^{(\alpha)}\right\}$, and, from the frequency of occurrence of every set, we estimated the probability, $P\left(N_{1}, N_{2}, \ldots, N_{m}\right)$, to observe the macroscopic state $\left(N_{1}, N_{2}, \ldots, N_{m}\right)$. To take into account only the relevant states, i.e. to neglect the configurations with very small probability, we compute the entropy

$$
\begin{array}{r}
S(N)=-\sum_{\left(N_{1}, N_{2}, \ldots, N_{m}\right)} P_{N}\left(N_{1}, N_{2}, \ldots, N_{m}\right) \times \\
\ln P_{N}\left(N_{1}, N_{2}, \ldots, N_{m}\right)
\end{array}
$$

from which we extract the number of macroscopic configurations with non negligible probability

$$
\mathcal{N}(N)=e^{S(N)} .
$$

A clusterization of $N$ elements in the language of number theory is a partition of the integer $N$, i.e., a possible way to obtain $N$ by summing integers - not necessarily all different. An upper bound for $S(N)$ and a guess for how it scales with the system size can be obtained noting that the asymptotic estimate for the number of partitions of $N, \mathcal{N}_{p}(N)$, is [8]

$$
\mathcal{N}_{p}(N) \simeq \frac{e^{\pi \sqrt{2 N / 3}}}{4 N \sqrt{3}} .
$$

From this we may guess that

$$
S(N)^{2} \simeq c(a) N+\text { cost }
$$

with $c(a) \leq c_{\max }=(2 / 3) \pi^{2} \simeq 6.58$. The constant $c(a)$ may be thought as an indicator of the fraction of the "active" or "relevant" elements in the system. In fact, if one defines the number $N_{a c t}$ by the relation

$$
\mathcal{N}(N) \sim e^{\pi \sqrt{2 N_{a c t} / 3}}
$$

it readily follows that

$$
\frac{c(a)}{c_{\max }}=\frac{N_{a c t}}{N} .
$$

In Fig. 1 1 we display $S(N)^{2}$ as a function of $N$ for different values of $a$. For each initial condition we checked that the cluster structure, found at the end of the thermalization, is a "permanent" structure, that was exactly the same after $6 \times 10^{4}$ and $1.2 \times 10^{5}$ successive iterations.

Figure 2 shows $c$ and the mean value $\langle\lambda\rangle$ of the maximum Lyapunov exponent of the system averaged over the initial conditions. The bars on $\langle\lambda\rangle$ indicate the variance $\sigma_{N}$. For $a>1.63$ the variance $\sigma_{N}$ goes to zero as $N$ increases and the probability distribution of the Lyapunov exponents, $P_{N}(\lambda)$, approaches a delta function, around some $\lambda>0$, as $N \rightarrow \infty$. On the other hand, for $a<1.63$ and for $N \rightarrow \infty \sigma_{N}$ approaches a non zero value, while $P_{N}(\lambda)$ approaches a nontrivial asymptotic distribution.

It is evident that for $a$ in the range $1.5 \div 1.7$, the system has a very large number of macroscopic states, while outside this range one detects only very few macroscopic states. We stress that we observe many macroscopic states - both chaotic and regular - not only many microscopic states, as in Ref. [9] where, in a system of $N$ globally coupled nonlinear oscillators, $(N-1)$ ! stable limit cycles are found. Moreover, the maximum of $c(a)$ is attained in the region where $\langle\lambda\rangle$ changes sign.

The above results and the observation of the histograms of the maximum Lyapunov exponent, suggest the existence of four distinct regions in the behavior of the system. These are roughly equivalent to those identified by Kaneko. We stress, however, that whereas Kaneko's classification is based on microscopic considerations, ours follows from the observation of a macroscopic behavior. The four regions are:

1. $a<1.5$, standard regular motion, with $\langle\lambda\rangle<0$ and few macroscopic states.

2. $1.5<a<1.63$, "glassy" regular motion, i.e.: $\langle\lambda\rangle<0$ and many macroscopic states.

3. $1.63<a<1.70$, "glassy" chaotic motion, with $\langle\lambda\rangle>0$ and many macroscopic states.

4. $a>1.70$, standard chaotic motion, with $\langle\lambda\rangle>0$ and few macroscopic states.

The analogy with the glassy behavior in disordered systems is not limited to the number of macroscopic states: in fact, in its glassy phase, system (1) is characterized by a violation of the selfaveraging. Given an initial condition, $\mathbf{x}(0)$, we may compute the time average, over a time $T$, of the variable $x_{i}$ :

$$
\left\langle x_{i}\right\rangle_{T}=\frac{1}{T} \sum_{n=1}^{T} x_{i}(n)
$$

or of any other function of a single element. In a standard situation, the probability distribution of $\left\langle x_{i}\right\rangle_{T}$ is expected to collapse to a delta function as $T$ and $N$ increase, that is, we expect that the time average over a very long time record of one element chosen at random inside a large system gives a complete description of the one-variable properties of the system.

Figure 3a shows the probability distribution of $\left\langle x_{i}\right\rangle_{T}$, obtained by gathering the average values for different $i$ and different initial conditions, in the phase with standard chaos. By increasing the observation time $T$, the variance decreases and one recover the usual behavior. 
Figure $3 \mathrm{~b}$ shows the situation in the case of the chaotic glassy phase: the increasing of $T$ does not modify the nontrivial structure of the distribution. A similar behavior is observed in the regular glassy phase.

These results, which are size independent, show that, in the glassy phase, the ergodicity is broken and the selfaveraging property does not hold, in other words: the time average on a single element is not sufficient for a complete characterization of the one-element statistics.

We conclude with a brief discussion about the role of noise. Of course, for finite values of $N$, the presence of noise will restore ergodicity after a very long time, which depends on $N$. Therefore it is interesting to consider the behavior of the system subject to noise on large but finite times. In Ref. [9] for large $N$ even a small amount of noise is sufficient to induce transitions among the different attractors. On the contrary we present a different picture. Within our approach, the scenario described above for the system (11) is very robust with respect to noise. In fact, the inclusion of a noise term $\sigma \eta_{i}(n)$ - where $\eta_{i}$ are independent random variables uniformly distributed in the interval $[-1,1]-$ to the r.h.s. of equation (1) does not change the probability distributions of $\left\langle x_{i}\right\rangle_{T}$ showed in Fig. 3a for noises strength as large as $\sigma \approx 10^{-2}$. Therefore the macroscopic states are stable for long times. This feature closely resemble to the behavior observed in glassy systems below the glassy transition temperature.

In conclusion we have shown that even simple models which do not contain any intrinsic disorder can have an extremely rich behavior at a macroscopic level. A behavior typical, e.g., of glasses. This scenario is very robust with respect to small external noise, indicating a "genuine" property of the system.

\section{ACKNOWLEDGMENTS}

We thank U. Marini Bettolo Marconi for useful discussions and for critical reading of the manuscript. (a) also INFN, Sezione di Roma 1

[1] W. Götze and L. Sjögren, Rep. Prog. Phys. 55, 241 (1992); M. Mezard, G. Parisi and M.A. Virasoro, Spin-Glasses Theory and Beyond (World Scientific, 1987); K.H. Fischer and J.A. Hertz, Spin-Glasses (Cambridge University Press, 1991)

[2] A review of aging in spin glasses and other disordered systems can be found in E. Vincent, J. Hammann and M. Ocio, in Recent Progress in Random Magnets, edited by D.H. Ryan (World Scientific, Singapore, 1992), Chap. 7, pp. 207-236.

[3] J.P. Bouchaud and M. Mézard, J. Phys. I (France), 4, 1109 (1994); E. Marinari, G. Parisi and F. Ritort, Report No. cond-mat/9406074 (unpublished); J. Kisker, H. Rieger and M. Schreckenberg, J. Phys. A 27, L853 (1994).

[4] Theory and applications of coupled map lattices, K. Kaneko (Ed.), (Wiley and Sons, Chichester, 1993)

[5] G. Parisi, Talk given at Thinking Science for Theaching: The Case of Physics, Report No. cond-mat/9412018 (unpublished).

[6] K. Kaneko, Phys. Rev. Lett. 63, 219 (1989)

K. Kaneko, Physica D55, 368 (1992).

[7] K. Kaneko, J. Phys. A24, 2107 (1991).

[8] K. Goldberg, M. Newman and E. Haynsworth, in Handbook of Mathematical Function, M. Abramowitz and I. A. Stegun Ed.s (Dover Publications, New York, 1970)

[9] K. Wiesenfeld and P. Hadley Phys. Rev. Lett. 62, 1335 (1989).

FIG. 1. Squared entropy $S^{2}$ as a function of the number of globally interacting elements $N$, for values of the parameter $a$ equal to $1.50,1.55$ and 1.63 . The lines are the linear best fits.

FIG. 2. Entropy coefficient $c$ (boxes) and average Lyapunov exponent $\langle\lambda\rangle$ (triangles) as functions of $a$. The values of $\langle\lambda\rangle$ and of $\sigma_{N}$ are computed with $N$ ranging from 100 up to 3200 , and with $\mathcal{M}=10^{4}$ initial conditions.

FIG. 3. Probability distribution of $\left\langle x_{i}\right\rangle_{T}$, at various observation times $T$ in the region of standard chaos $-a=1.80-(\mathrm{a})$ and in the region of glassy chaos $-a=1.67-(\mathrm{b})$. The different curves correspond to averages performed, after a transient of $6 \times 10^{4}$ steps, on time intervals $T=0.75 \times 10^{4}, 1.5 \times 10^{4}$, $3 \times 10^{4}, 6 \times 10^{4}, 12 \times 10^{4}$. For each curve $\mathcal{M}=10^{3}$ initial conditions were considered with $N=300$. The histograms do not change qualitatively by changing $N$ up to 1200 . 


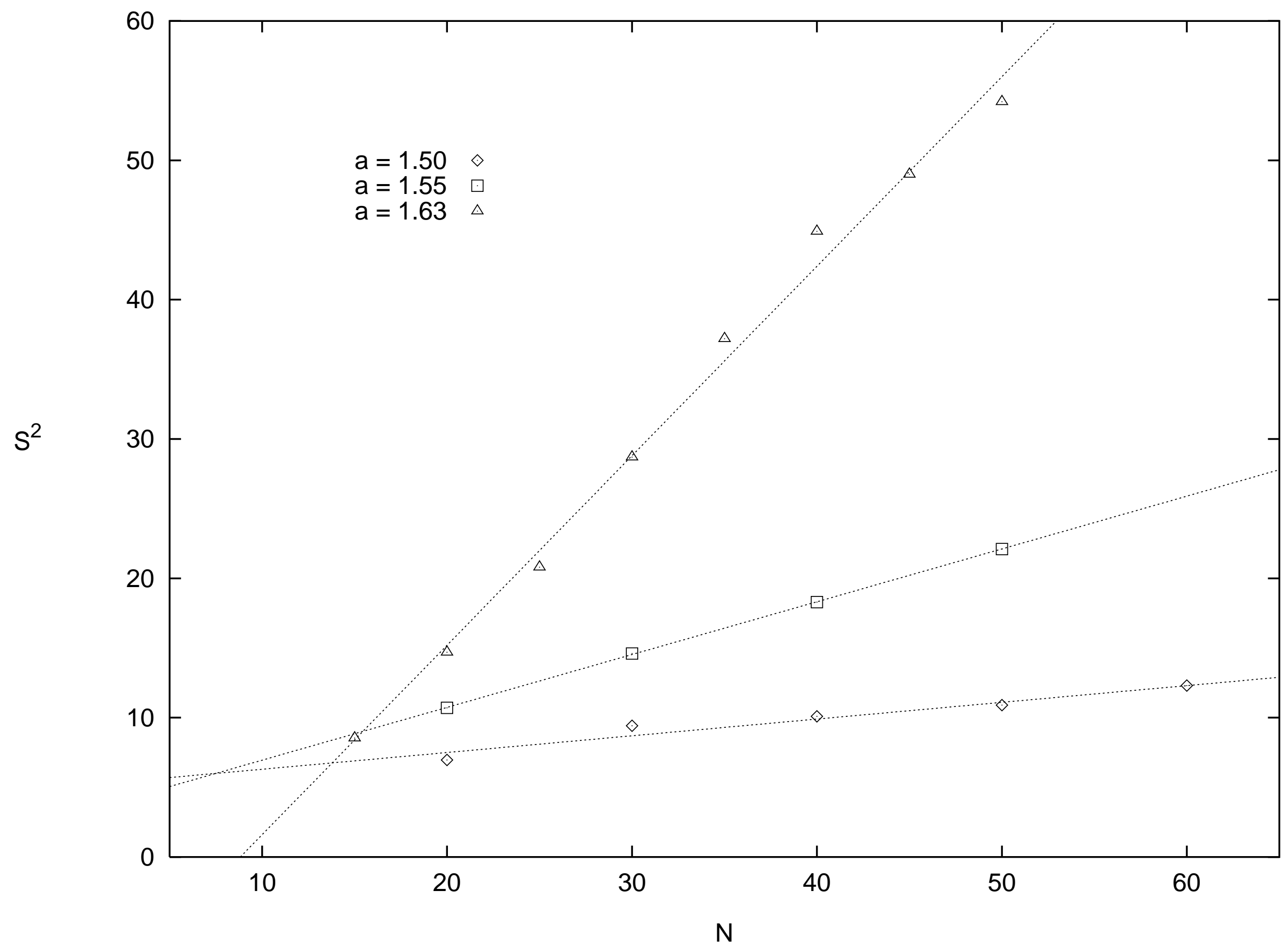




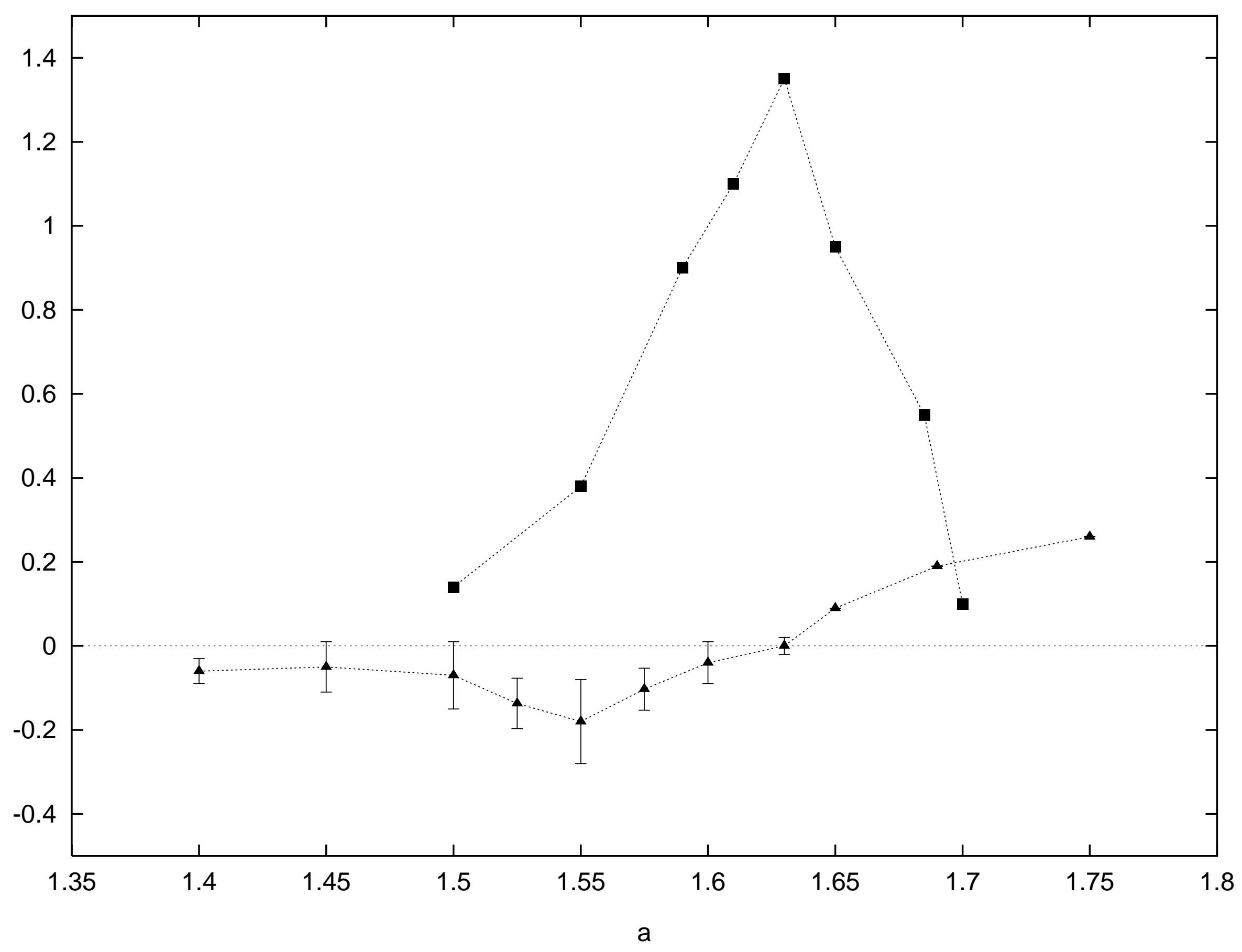




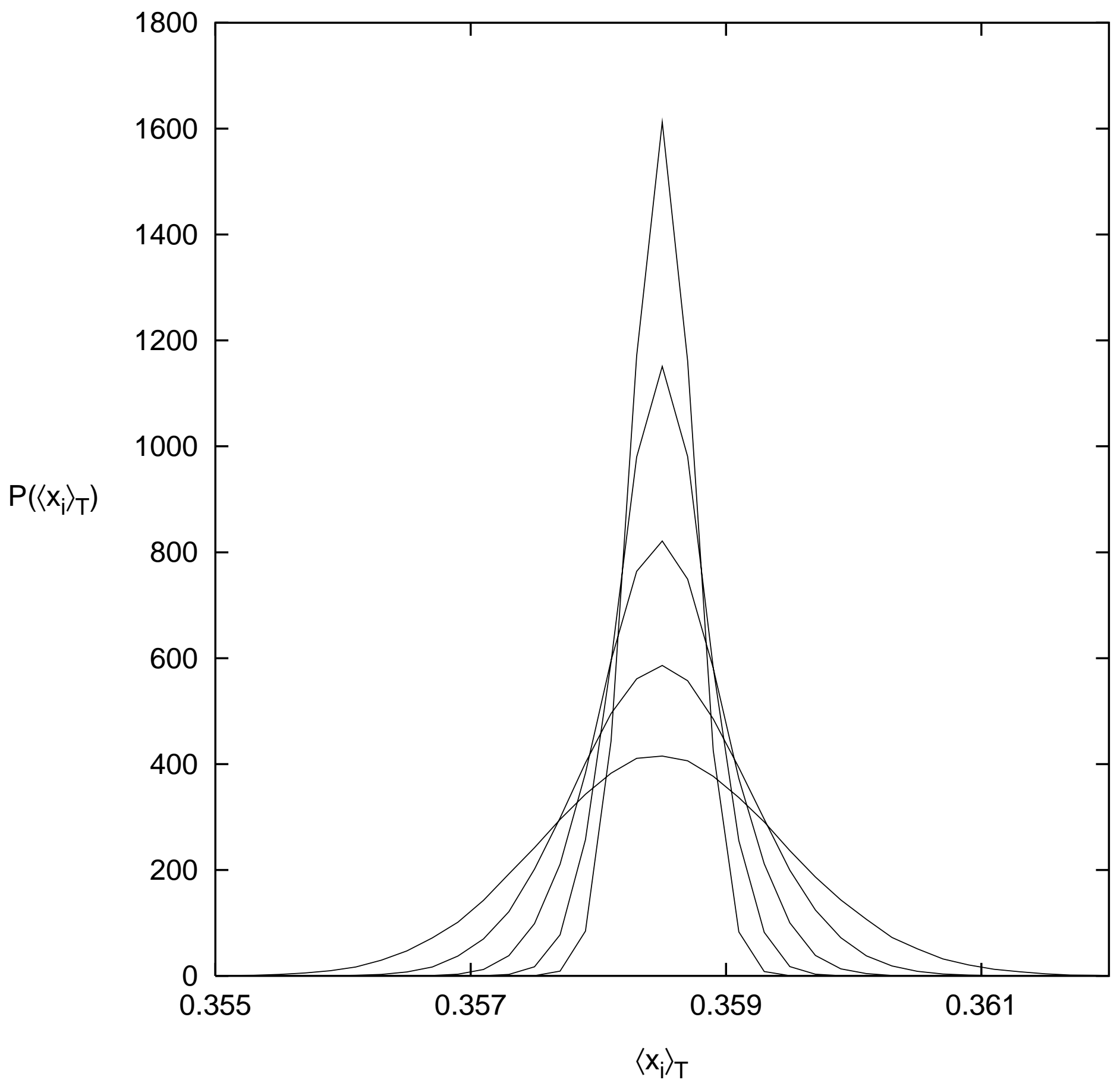




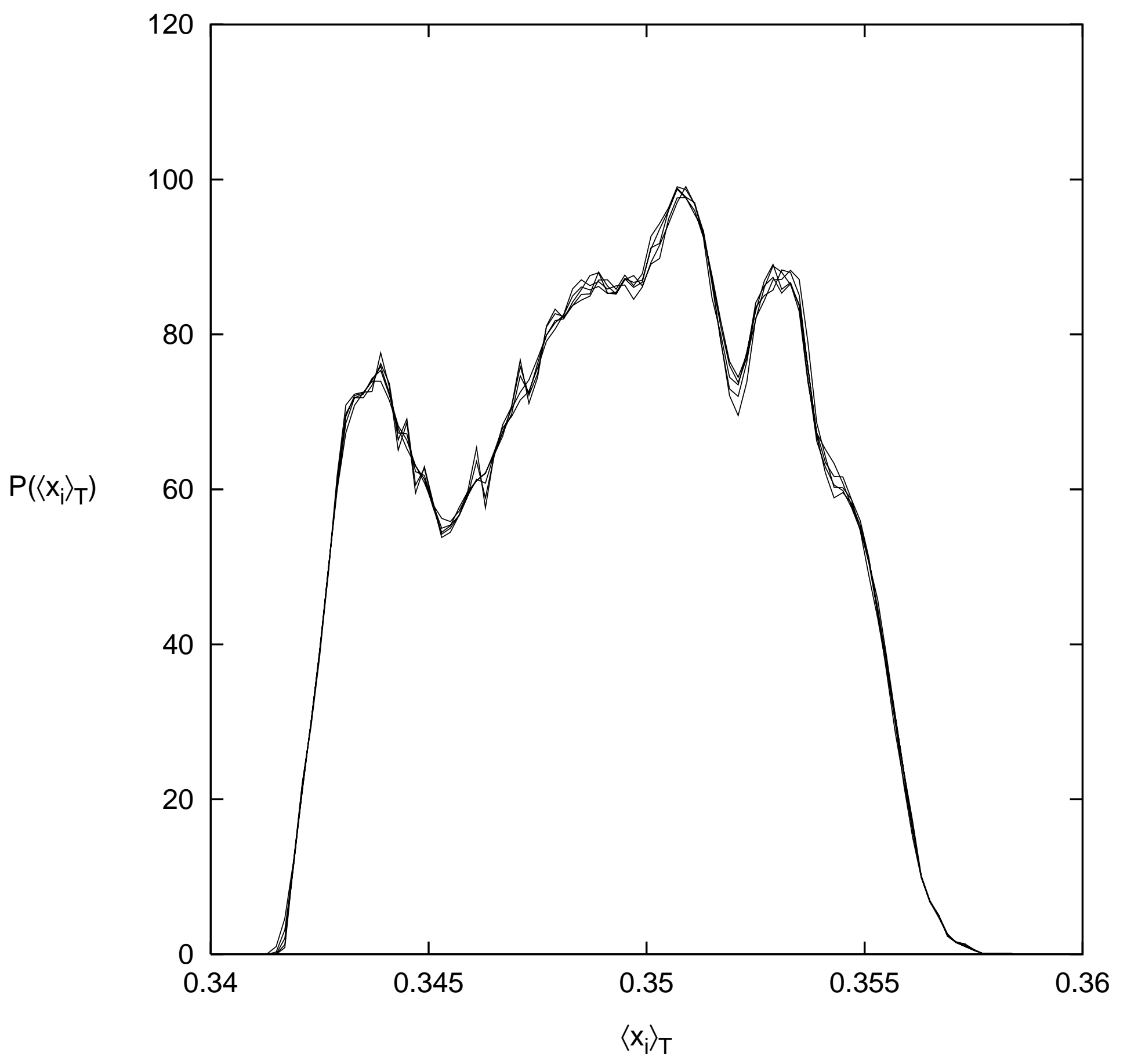

